\title{
Nanosecond laser-induced interference grating formation on Silicon
}

R. J. Peláez ${ }^{1,2}$, E. Rebollar ${ }^{3}$, R. Serna ${ }^{1}$, C. Acosta-Zepeda ${ }^{4}$, P. Saavedra ${ }^{5}$, J. Bonse ${ }^{6}$, E. Haro-

Poniatowski ${ }^{1,4}$

${ }^{1}$ Laser Processing Group, Instituto de Óptica, Consejo Superior de Investigaciones Científicas, Serrano 121, 28006 Madrid, Spain

${ }^{2}$ Instituto de Estructura de la Materia, Consejo Superior de Investigaciones Científicas, Serrano 121-123, 28006 Madrid, Spain

${ }^{3}$ Instituto de Química Física Rocasolano, Consejo Superior de Investigaciones Científicas, Serrano 119, 28006 Madrid, Spain

4 Departamento de Física, ${ }^{5}$ Departamento de Matemáticas, Universidad Autónoma Metropolitana Iztapalapa, Av. San Rafael Atlixco No. 186, Col. Vicentina, C.P. 09340, México D. F. México

${ }^{6}$ Bundesanstalt für Materialforschung und -prüfung (BAM), Unter den Eichen 87, 12205 Berlin,

Germany.

Abstract:

The formation of gratings on the surface of a silicon wafer by nanosecond laser irradiation through a phase mask using an ArF laser emitting at $193 \mathrm{~nm}$ is studied. The phase mask along with some focusing optics is capable to generate via interference a periodic intensity distribution, which can be used for surface patterning. The surface patterning strongly depends on the laser energy density and on the number of pulses, as revealed by Atomic Force Microscopy (AFM). The results show that irradiation even with a single laser pulse produces periodic depth modulations on the surface. The spatial surface modulation is in the micrometer $(1.7 \mu \mathrm{m})$ range while the depth modulation is in the nanometer regime $(1-20 \mathrm{~nm})$. With increasing number of pulses (1-100) the depth modulation amplitude increases smoothly. Increasing the number of pulses further results in the progressive destruction of the grating, vanishing completely after $\sim 5000$ pulses. This evolution is also monitored in-situ by measuring the intensity of the first order-diffracted probe beam and the behavior is in accordance with what is observed by AFM. Finally, we qualitatively explain the results invoking thermally 
induced effects in the melted $\mathrm{Si}$ : these physical processes involved are probably thermocapillary and/or Marangoni effects inducing material displacement as the surface melts. 


\section{Introduction}

Silicon has been the most commonly used semiconductor material for many years, and periodic micro- and nanostructures fabricated on it may play an important role in applications in different fields. In fact, in modern technologies such as the fabrication of solar cells or optoelectronic devices [1-3] texturing of silicon is a critical process. There are several techniques for micro- and nanostructuring silicon. Among them, the most common ones are based on lithographic methods [4,5], nanoimprinting [6], chemical $[7,8]$ and reactive ion etching [5], and laser techniques [3,9,10].

It is well known that irradiating a surface of a solid by laser pulses can have a wide variety of effects. In the late seventies laser annealing was developed in order to recrystallize ion-implanted semiconductors and in particular silicon. This laser annealing process typically takes place in the nanosecond (ns) regime. At this scale of time the models to explain the observations are based on thermal effects [11]. Since the development of shorter laser pulses the subject has been in constant investigation in the single-pulse and multi-pulse regimes. Interesting results have been obtained in the ps- and fs-time domains [12]. Also, as

early as 1965, M. Birnbaum observed periodic ripples formation by laser irradiation [13]. Another important breakthrough of particular interest has been the development of "black silicon", where hierarchical micro-/nanostructures feature enhanced optical absorption in solar cells or have important applications as hydrophobic surfaces.

Laser-induced surface patterning of silicon has been the subject of several studies in recent years. By direct irradiation with nanosecond [14,15] and femtosecond pulses [16,3], irradiation through a hole [17] or using arrays of self-assembled micro- and nanospheres [18,19], formation of laser induced periodic surface structures (LIPSS) [20] and laser-induced interference lithography (LIL) with up to four beams [21,22].

In this work, the formation and evolution of a pulsed laser-induced grating is monitored ex-situ by AFM in the surface of a silicon wafer as a function of the number of pulses. It is found that even with a single nslaser pulse a spatial modulation with a periodicity in the micrometer range and a depth of the order of one nanometer is generated. This evolution can also be monitored insitu by measuring the intensity of the first order-diffracted continuous wave (cw) probe beam. According to our experimental results the physical processes involved in the grating formation are related with a thermocapillary effects inducing material displacement as the surface melts. 


\section{Experimental}

The samples are p-type single-crystal Si wafers with $\langle 100\rangle \pm 1$ deg orientation and $4-70 \mathrm{ohm}$ cm resistivity held at room temperature, cleaned with acetone and ethylic alcohol ultrasonic bath and dried with dry nitrogen. Figure 1 shows the experimental set-up used to irradiate the samples by the technique of phase mask laser interference. Pulses of an $\operatorname{ArF}$ excimer laser $(\lambda=193 \mathrm{~nm}, \tau=20 \mathrm{~ns})$, that pass through the square-shaped aperture, are used to illuminate a fringed phase mask composed by parallel lines, parallel to the $y$-axis, with a period of $20 \mu \mathrm{m}$. It is optimized for high efficiency (> 90\% of fluence) in the $\pm 1 \mathrm{st}$ diffraction orders at $193 \mathrm{~nm}$ and it is manufactured by Laser Laboratory, Göttingen, Germany, on SiO2 plates [23].

These two diffraction orders are forced to interfere at the Si surface using a couple of lenses (L1, L2) in a telescope configuration. Thus, the Si surface is exposed to a modulated intensity formed by the maxima and minima of interference $[24,25]$. The local energy density (fluence) distribution is modulated along the direction perpendicular to the fringes ( $x$-axis) as $f(x)=F(1+\cos (2 \pi \cdot x / \Delta))$ where $F$ is the average fluence measured at the sample site. It will be referred to from now on as fluence and it is in the range $440-1000$ $\mathrm{mJ} \mathrm{cm}-2$. The spatial period, $\Delta$, at the sample site is $\Delta=1.7 \mu \mathrm{m}$, and the laser beam spot size is $0.4 \mathrm{x} 0.48$ mm2. The number of accumulated laser pulses (\#) for each spot is in the range $1-5000$ pulses. The repetition rate of the ArF laser pulses is $1 \mathrm{~Hz}$ when the number of total laser pulses is lower than 50 and it operates at $5 \mathrm{~Hz}$ for higher number of laser pulses. The sample is mounted on a $X Y$-translation stage with micrometer resolution. We have produced more than 25 surface irradiations changing the number of laser pulses and the laser fluence.

A continuous wave $(\mathrm{cw})$ Helium-Neon $(\mathrm{He}-\mathrm{Ne})$ laser beam $(\lambda=632.8 \mathrm{~nm})$ and two photodiodes are used to record in-situ the evolution of the zero and first diffraction order of the irradiated surface in reflection mode. The incident He-Ne laser beam (p-polarized) is in the plane (ZY) normal to the sample surface $(X Y)$ and parallel to the fringe patterns direction (y-axis) and it is focused by a lens (L) at the center of the irradiated area at $45^{\circ}$ of their normal. Thus, the diameter of the probe beam on the sample surface is $\leq 200$ $\mu \mathrm{m}$. The surface topographies of some irradiated regions are characterized with an atomic force microscope AFM (Bruker Multimode 8, with a Nanoscope V controller) operated in tapping mode using Tap300Al-G 
probes (budgetsensors). Surface patterns generated with the highest number of laser pulses are examined by field emission scanning electron microscopy (FE-SEM) in a Hitachi SU-8000 microscope in crosssection view. Finally, the surface patterns are analyzed by using an optical microscope (Nikon Eclipse Ti) operating in reflection bright field configuration at $\lambda=455 \mathrm{~nm}$. Reflectivity images have been calibrated using a non-irradiated $\mathrm{Si}$ wafer, thus the final images represent the normalized surface reflectivity change relative (in percentage) to the crystalline $\mathrm{Si}$ and defined as $\mathrm{Rirr}=100 \cdot((\operatorname{Iirr}-B) /(I \mathrm{Si}-B))$, where $I S i$ and Iirr correspond to the images of the nonirradiated Si wafer and irradiated region respectively and $B$ is the background signal image of the camera system.

\section{Results and discussion}

Figure 2 shows three photos of the same Si sample illuminated with natural white light at different angles of view. The colored areas correspond to the regions irradiated with the laser interference technique and the dark gray regions between the areas correspond to non-irradiated zones on the Si sample. The angle between the normal at the sample surface and camera position is $\theta \approx \sin ! !(\lambda / \Delta)$, which is the grating equation for the first diffraction order in normal illumination. Thus, the colors correspond to the light diffracted by the first diffraction order of the periodic grating structures. The observation angle, $\theta$, is increased slightly from the left to the right images, thus the diffracted color shifts from blue to red (higher wavelengths) according with the above equation. Three columns of square-shaped areas are clearly visible for each photo, with the fluence and the number of laser pulses of each area being different. The number of laser pulses, \#, increases from 250 to 2500 from top to bottom and the laser fluence, $F$, increases from the left to the right column from 630 to $1000 \mathrm{~mJ} \mathrm{~cm}-2$. It is worth noting that the irradiations with the minimum fluence $(440 \mathrm{~mJ} \mathrm{~cm}-2)$ do not produce any detectable signal after 2000 laser pulses. This result clearly shows that this value is below the threshold fluence of material transformation. However, the irradiations at $F \geq 630 \mathrm{~mJ} \mathrm{~cm}-2$ produce a visible and detectable optical diffraction. Precise time-resolved reflectivity measurements of ArF excimer irradiations on c-Si showed that the single-pulse melting threshold of c-Si is $650 \mathrm{~mJ} \mathrm{~cm}-2$ [26], which is slightly higher than the transformation fluence threshold found in this work $(<630 \mathrm{~mJ} \mathrm{~cm}-2)$. It is remarkable that this last parameter corresponds to the average fluence, $F$, that is modulated between minima $(f(\Delta / 2)=0)$ and maxima of interference $(f 0=2 F)$, according to the expression described in the experimental section. Therefore, the melting threshold is easily reached at the maxima of 
interference for $F=630 \mathrm{~mJ} \mathrm{~cm}-2$. According to the spatial fluence distribution at the sample surface described in the experimental section, the materials transformation threshold should be locally exceeded in the case of $F=440 \mathrm{~mJ} \mathrm{~cm}-2$ at $x=0$ and $x=\Delta$, where $F(x)=2 F$. Apparently, the heat diffusion from hot to cold region plays an essential role in this case preventing the formation of a permanent surface relief. This argument is confirmed by the following analysis of the melting threshold fluence and the thermal diffusion length accompanying the laser irradiation.

We have recently used a heat diffusion model C. Acosta-Zepeda et al. [27] in order to theoretically investigate the induced modifications on a Si surface by single pulse nanosecond laser irradiation. This model solves the Stefan boundary value problem from the differential equation for heat conduction:

$$
\rho c_{p} \frac{\partial T(z, t)}{\partial t}-\frac{\partial}{\partial z}\left(K \frac{\partial T(z, t)}{\partial z}\right)=P(z, t)
$$

where $\rho$ is the mass density, $c \mathrm{p}$ the specific heat, $K$ the thermal conductivity and $P(z, t)$ the heat generation function. To determine the melting fluence threshold with the equation above, the fluence for which the temperature $T(z, t)$ reaches the melting point is computed. This study has been done considering different irradiation wavelengths at different laser fluences. The melting threshold fluence at a wavelength of 193 $\mathrm{nm}$ and for a pulse duration of $20 \mathrm{~ns}$ is found to be $F \mathrm{~m}=440 \mathrm{~mJ} / \mathrm{cm} 2$, very close to the experimental values reported before.

The thermal diffusion length, $L t h$, in Si for the pulse duration, $\tau$, depends on the thermal diffusivity $D=$ $0.86 \mathrm{~cm} 2 / \mathrm{s}$ at $T=300 \mathrm{~K}$ [28] according to $L$ th $=(D \tau)^{1 / 2}$, resulting in a value of $1.31 \mu \mathrm{m}$ which is higher than $\Delta / 2=0.85 \mu \mathrm{m}$. If the period approaches the thermal diffusion length, the temperature difference between maxima and minima intensity positions decreases and does not give rise to a periodic melting surface, but it leads to a more homogeneous heating source. Therefore, the diffusion of heat from hot to cold surface regions occurs during the ns laser pulse and the temperatures at the maximum and minimum of interference are expected to be balanced at the end of the laser pulse. Thus, temperature at the maxima of interference for $F=440 \mathrm{~mJ} \mathrm{~cm}-2$ does not reach the melting temperature of $\mathrm{Si}$ (1681 K [29]).

Figure 3 presents the pulse number (\#) dependence of the photodetector signal of the first-order diffracted $\mathrm{cw}$-laser beam at patterned regions generated at three different laser fluences accounting to 630,830 , and $1000 \mathrm{~mJ} \mathrm{~cm}-2$, respectively. Figure $3 \mathrm{a}$ shows that the signals follow at all different fluences a similar trend; 
the diffraction efficiency monotonically increases approximately from the first pulse up to $2500-3000$ pulses. For a higher number of pulses, the signal decreases again toward values close to zero. In addition, the diffraction signal increases with fluence for $\# \lesssim 2750$. In this region, where $0<\# \lesssim 2750$, we can distinguish different stages in the evolution of the diffracted signal. The detailed behavior during the first pulses (Fig. 3b), shows clearly three different slopes. Diffraction increases very rapidly from the initial zero level from the first pulse up to \# 100 - 200 pulses (depending on $F$ ) and after that it increases but at lower rate, and then signal increases significantly when $\# \geq 1500$ - 2000 pulses.

In summary, signals acquired at different $F$ shows the same general trends of the diffraction efficiency in the first diffraction order and four different stages (I - IV) are clearly distinguished according with the evolution of the signal with \#: increases at high rate for $1 \lesssim \# \lesssim 100-200$ (stage I), increases at low rate for \# $\lesssim 1500$ - 2000 (stage II), increases at high rate for \# $\lesssim 2500-3000$ (stage III), and finally decreases at high rate for \# $\lesssim 4000$ (stage IV). The full circles in Figs. 3a and 3b mark approximately the borders between stages I/II, II/III, and III/IV. Fig. 3c shows the pulse number span of the four different stages according with the position of the full circles marked in Fig. 3a and 3b. The pulse number span of stages I and II increases with $F$ and that of stage IV decreases with $F$, while the span of stage III does not significantly depend on $F$. However, from spot to spot the effects could vary due to variations in the intensity and spatial profile of the laser from pulse to pulse.

Figure 4 shows the optical micrographs of the center of the patterned region produced at the highest laser fluence of $1000 \mathrm{~mJ} \mathrm{~cm}-2$. The labels correspond to the number of laser pulses used for exposure. A periodic reflectivity modulation in the horizontal direction ( $x$-axis) is clearly visible in all images. A detailed examination shows that for \# = 1 some thin and weak dark fringes are present in the vertical direction. This is in complete agreement with the small but detectable signal measured in the first diffraction order. All the images show these dark fringes that are vertically aligned on purpose and whose width, darkness/brightness and shape depends on \#.

Figure 5a shows the reflectivity profiles of the fringes that are the average of the images shown in Fig. 4 along the fringe direction in a single representative period. Figure $5 b$ shows the corresponding profiles arranged in a two-dimensional (2D) map. The surface reflectivity is normalized to that of the non-irradiated region (crystalline $\mathrm{Si}$ ) accounting to $100 \%$ here, see definition of Rirr in the experimental section. However, 
the absolute values of reflectivity and the profile shapes must be handled with caution because the images shown in Fig. 4 have been acquired with the maximal magnification of the microscope and some details may have been blurred since they are smaller than the optical resolution of the microscope. The optical reflectivity, in percentage, is close but below $100 \%$ for $\# \leq 100$ (stage I) and it increases progressively at the center of the period as \# increases. The reflectivity increases rapidly beyond $100 \%$ as \# increases for \# $>100$ and it reaches $102 \%$ at the maximum \# analyzed (end of stage III). It is remarkable that this maximum of relative reflectivity appears at $x \sim \Delta / 3$ instead of $x=\Delta / 2$ which correspond to the middle position between dark fringes. This asymmetry is clearly visible for the highest \# and it is less visible for lower \#, being the position of the maximum of reflectivity at $x \sim 0.42 \Delta$ and $x \sim 0.45 \Delta$ for $\#=1000$ and $\#=500$, respectively. Thus, the asymmetry of the maximum of reflectivity has been clearly identified in the stages II and III and it increases as \# increases.

Figure 6 provides AFM topography images of surface patterns that have been exposed to a different number of laser pulses up to 1500 . Herein, the fringes are clearly visible as periodic surface modulations with heights less than $15 \mathrm{~nm}$, arranged in the vertical direction for a varying number of pulses.

Depth profiles of a representative period of the AFM images corresponding to irradiation with different \# are plotted in Fig. 7a. The profiles evolve from a small elevation/depression for low \# to a well-defined protrusion/hole for larger \# and the shapes and heights of these surface features also depend on \#. Figure $7 \mathrm{~b}$ shows the $2 \mathrm{D}$ arrangement of theses profiles, where the horizontal axis represents the position along one period and the vertical axis displays the number of laser pulses accumulated in the irradiated region. The colors correspond to the topographic height (in $\mathrm{nm}$ ) relative to the line average value set as zero. Moreover, some AFM images are acquired at the border of the irradiations where the local irradiation fluence drops to zero (data not shown here). The transition zone from the non-irradiated regions to the irradiated regions, where the first fringes are produced, shows the height/mass conservation between them; i.e., the amount of Si evaporated is not significant and the laser irradiation produces just a mass transport (lateral displacement) of Si. This result is in close accordance with what was reported before for the case of Si irradiated through a pinhole [17]. For that reason, we have assumed that the amount of Si must be preserved in a period, and thus the average height in the horizontal direction in Fig. $7 \mathrm{~b}$ has been forced to be zero. 
If we define $z$ as the difference between the minimum depth and maximum height for each profile, it evolves from $3 \mathrm{~nm}$ to $20 \mathrm{~nm}$ as \# increases (Fig. 8a). According to the four evolution stages defined previously, $z$ increases progressively from $3 \mathrm{~nm}$ up to $\sim 10 \mathrm{~nm}$ in the stage I, subsequently it varies in the range $10-15 \mathrm{~nm}$ in the stage II without a clear dependence on \#, and it increases up to approx. $20 \mathrm{~nm}$ in the stage III. This evolution is clearly visible in Fig. 7b, where the maximum height increase in stage I, is approximately constant in stage II and afterwards increases rapidly in stage III. In Fig. 8a the evolution of the first order diffracted signal for $F=1000 \mathrm{~mJ} \mathrm{~cm}-2$ (black solid line in Fig. 3a) and the optical contrast, $C$ in percentage, defined as the difference between the Rirr of the bright and dark regions of the profiles shown in Fig. 5b, are also included.

Figure $8 \mathrm{~b}$ shows the full width at half maximum (FWHM) and the asymmetry of the AFM profiles, $A$, defined as the peak $x$-position of the profiles shown in the Fig. 7, where $A=0.5 \Delta$ corresponds to peaks placed at the center of the period and the asymmetry of the profile increases when the parameter $A$ is far away from that value. On one hand the FWHM is in the range $1000 \pm 70 \mathrm{~nm}$ for $\# \leq 100$ pulses (except for the first pulse, where this estimation has the highest uncertainty) and afterwards it decreases to $660 \mathrm{~nm}$ for \# = 750. For higher \#, the FWHM increases to $920 \mathrm{~nm}$ again. On the other hand, the profile is approximately symmetric $(0.48 \Delta<A<0.51 \Delta)$ for $\#<100$ pulses and the asymmetry varies from $A=0.44 \Delta$ to $A=0.35$ $\Delta$ as \# is increased from 100 pulses to 1500 pulses, and finally the profile is clearly asymmetric $A=0.37 \Delta$ for \# = 2500 pulses. In summary, the height/depth of the broad and symmetric profiles increases with \# in the stage I. Their width decreases and becomes asymmetric with \# in the stage II, and finally the profile is narrow and asymmetric in the stage III where it reaches its maximum height/depth.

The contour of the surface profile that is periodically repeated modifies the intensity of the diffraction signal. In order to analyze this relation, we have calculated the diffraction efficiency by rigorous coupledwave theory implemented using the software package GD-Calc ${ }^{\circledR}$ Version 06/13/2017 [30]. This program allows us to calculate the diffraction efficiency of uniperiodic gratings with a defined profile, which is defined by 50 stacked lamellar strata. Lengths and heights of these strata determine the contour of the periodic profiles, which have been adjusted in order to obtain profiles with the values of depth/height $(z)$, width $(F W H M)$ and asymmetry $(A)$ determined experimentally and shown in Figs. 8a, and 8 b. The diffraction efficiency in the first diffraction order in reflection mode is then calculated for each surface 
profile for the angle of incidence and laser beam polarization defined in the experimental section. These calculated values together with the experimental diffraction signal are plotted in Fig. 8c. Results show that the calculated diffraction efficiency depends strongly on the height/depth ratio of the profile while the asymmetry and width become less relevant. These estimations are in excellent agreement with the experimental diffraction signal, and the stages I, II and III, defined previously in the Fig. 8 a, are identifiable in these calculations as well. The diffraction efficiency monotonically increases in stage I, it is approximately constant in stage II, and increases rapidly in stage III. These results support the conclusion that the diffraction signal is mainly related to the surface topography and here is not related to the possible structural modification of the $\mathrm{Si}$ due to the laser irradiations affecting the optical constants of the material. Taking into account all the previous results, we can consider that matter transport processes arise from the Marangoni effect, with thermocapillary forces acting on the molten silicon - driven by local temperature differences. First, the absorption coefficient $\alpha$ of silicon is around $1.6 \cdot 106 \mathrm{~cm}^{-1}$ at $193 \mathrm{~nm}$ [31] and thus, radiation is absorbed in a thin near surface layer of around $6 \mathrm{~nm}$ thickness, according to the optical penetration depth $1 / \alpha$. Melting of silicon by nanosecond pulsed lasers has been widely studied, and the melting depth and melting duration have been reported to be up to 200 or $600 \mathrm{~nm}$ and up to 70 or $170 \mathrm{~ns}$ for irradiation at $193 \mathrm{~nm}$ with pulses of $20 \mathrm{~ns}$ and fluences of 1 or $2 \mathrm{~J} \cdot \mathrm{cm}-2$ respectively [11]. As the period of the microstructures has a value close to the thermal diffusion length, the temperature difference between maxima and minima intensity positions decreases and all the surface is melted. This produces a moderate modulation in the temperature field since the heat generated at the interference maximum position on the periodical intensity distribution dissipates to the interference minimum position during the laser interaction time. Thus, on the one hand almost all irradiated volume of silicon is having a high temperature above the melting point and is in the liquid form and on the other hand no evaporation of the material is induced because of high thermal losses in the hot maxima position due to thermal diffusion. This is in agreement with the results obtained by AFM; as mentioned, AFM at the border of the irradiated area shows that the amount of $\mathrm{Si}$ evaporated is not significant. In order to explain the grating formation without vaporization of the material, we must consider the Marangoni effect [21]: the molten silicon moves towards the low intensity regions. It is known that the surface tension of molten silicon depends on temperature 
[32]. In particular, it is lower in the interference maximum, which corresponds to a hotter area, and it is higher in the interference minimum, which corresponds to a colder area. Thus, molten silicon is dragged from interference maximum to minimum positions, where it encounters fluid moving in the opposite direction driven by same force from neighbored interference ridges. These liquids overlap at minimum of interference and raise the height in the $z$-direction.

The average velocity of the silicon liquid in the $x$-direction can be analyzed using the lubrication approximation, which describes the liquid motion due to the gradient of the surface tension (Marangoni effect) and the capillary effects on curved surfaces (Laplace effect) [10]. The former is proportional to the surface thermal gradient $(d \gamma / d T<0$ for $\mathrm{Si})$ and the latter is proportional to $(d 3 z / d x 3)$ but directed opposite to the Marangoni effect. As a first approximation, it can be assumed that the Marangoni effect dominates the liquid motion when the surface profile is not abrupt and the period to height ratio is $>20$ [10,33]. In our case this ratio is $>100$ in the region I and we could expect that the liquid movement is dominated by the Marangoni effect. Thus, if the thermal distribution, and consequently the liquid movement, is well reproduced pulse by pulse, the liquid is accumulated at the interference minima, and the profiles are symmetrically distributed, which would induce that the heights increase linearly with the number of pulses. If the laser fluence is reduced, the molten liquid, its lifetime and thus the height increased per pulse would be also reduced.

We have used the above mentioned model considering thermal heating, melting and subsequent melt flows by the Marangoni effect, C. Acosta-Zepeda et al. submitted [27], to compute the superficial topographic formation generated by a single laser pulse $(193 \mathrm{~nm}, 20 \mathrm{~ns})$ on crystalline Silicon. In this approximation we have used a spatial fluence distribution that includes two consecutive interference maxima of the function: $f x=F \cdot(1+\xi \cos (2 \pi \cdot x / \Delta))$, where $F=800 \mathrm{~mJ} / \mathrm{cm} 2$ is the average fluence measured at the sample site and $\xi=0.025$ is an adjustable parameter in order to avoid the fluence taking values below the melting threshold. The spatial period, $\Delta$ is set to the experimental value of $1.7 \mu \mathrm{m}$. The results are presented in the Figure 9 and compared to the surface topography observed experimentally.

The associated optical interference pattern at the sample surface has two maxima and one minimum (at $x=$ 0), which corresponds to one representative spatial period o. In Fig. 9 the calculated surface deformation (green line) and the experimental surface profile obtained by AFM (blue line) are compared. Although this 
model considers only the Marangoni effect it reproduces the experimental results quite reasonably. In stage II the modulated laser light interacts with a periodically corrugated surface. It generates scattered light that interferes with the incident laser radiation producing an additional light modulation at the sample surface. It causes a local enhancement of electromagnetic field at sharp features of the surface topography[10] and results in an asymmetric distribution of the laser light across the surface. In these conditions the displaced silicon melt parts do not meet at the interference minima as they have different properties, in terms of speed, temperature or volume. As a consequence, the center of mass is shifted away from the center of the period (interference minima), causing an asymmetric surface profile. These subtle surface profile asymmetries may be enhanced by the asymmetric distribution of absorbed optical energy imposed by the next laser pulse. Through this positive feedback mechanism, the asymmetry of the light modulation and surface profile are further amplified pulse by pulse.

Asymmetric surface profiles are characterized by the gradual and the abrupt surface slopes; the higher the asymmetry, the higher the abrupt slope. In the stage II the surface modulation height is approximately constant and the asymmetry is increased pulse by pulse, and thus the contribution of capillary effects to the melt displacement (proportional to $d^{3} z / d x^{3}$ ) increases pulse by pulse. Supposedly, this effect, which is opposite to the Marangoni effect, is not negligible in the abrupt side of the asymmetric profiles and the dynamics of the liquid wave is modified by this capillary effect. The competition of the Marangoni and capillary effects combined to the asymmetric light distribution may be responsible for the increase of the profile height and the diffraction signal in the stage III. This regime ends when the surface is damaged by the laser irradiation.

Figure 10 shows a top-view optical micrograph, an AFM topography image, and a SEM crosssectional view of the irradiations produced for $F=1000 \mathrm{~mJ} \mathrm{~cm}-2$ and \# = 5000 (stage IV). The optical reflectivity image shows a quasi-random distribution of dark points. The AFM topography shows that these black points correspond to protruding structures and SEM crosssection image shows a clear picture of these microcolumns of around $2 \mu \mathrm{m}$ height.

Similar column structures have been obtained by cumulative quasi-homogeneous intensity excimer nanosecond $(\mathrm{KrF} \lambda=248 \mathrm{~nm})$ pulsed irradiations at $3000 \mathrm{~mJ} \mathrm{~cm}-2$ in air [34], a specific surface morphology termed "black silicon" in the literature. It was suggested that inhomogeneous surface corrugations modified 
the local laser fluence absorbed due to an altered angle of incidence of the laser radiation, and therefore the ablation takes place at the minima and maxima of the surface. This inhomogeneous ablation produces a preferential mass transport through vapour that is re-deposited at surface protrusions. After several pulses protruding columns can be formed and their formation is reinforced by multiple reflections into the emergent column. In this experiment, the interference nature of the laser irradiation may enhance the inhomogeneous laser absorption and surface topography and thus may improve the $2 \mu \mathrm{m}$ production of microcolumns [35]. The growth of these structures breaks the periodicity of the fringes and thus diffraction efficiency goes to zero at the end of stage IV. In addition, this destruction of the periodic signal is accompanied by a decrease of the specular reflection (data not shown here). The corresponding specular reflectivity taken at the zero diffraction order with the probe pulse with the described configuration, $R$, is approximately constant in stages I, II and III, however at the end of stage IV the ratio $R / R O=0.82 \pm 0.02$ irrespective of the fluence, where $R O$ is the reflectivity of the non-irradiated (polished) Si. This surface reflectivity reduction is related with the texturization of the Si surface. Production of Si surfaces with low reflectivity has interest in the production of efficient solar cells based on multicrystalline Si, and many efforts have been expended investigating the production of this texturized Si [36,37]. For instance the nanosecond $\mathrm{KrF}$ laser irradiation of $\mathrm{Si}$ in air, in the mode of scanning at the fluence of $2200 \mathrm{~mJ} \mathrm{~cm}-2$ produces these types of microcones, or nanosecond irradiations with the second harmonic of a Q-switched Nd:YAG laser with a fluence of $500 \mathrm{~mJ} \mathrm{~cm}-2$ do not develop textures till the number of accumulated pulses reaches 104, and as the laser fluence reaches the ablation threshold $(\sim 2000 \mathrm{~mJ} \mathrm{~cm}-2$ at $\lambda=532 \mathrm{~nm})$ the texturization appears after the accumulation of 100-500 pulses and for higher values of fluences (3000 mJ $\mathrm{cm}-2)$ the spikes appear for 50 laser pulses [38]. ArF nanosecond excimer irradiation of $\mathrm{Si}$ in air produces micrometric columns (3-30 $\mu \mathrm{m}$ tall) after some hundreds of laser pulses at a fluence of $1500-2000 \mathrm{~mJ} \mathrm{~cm}-$ 2, and they observed noticeably modifications by melt process after some thousands of pulses at $700 \mathrm{~mJ}$ $\mathrm{cm}-2$. In the present study, the decrease of the surface reflectivity, and thus the texturization, appears in the range of $\sim 3500$ - 4000 pulses without any clear dependence on the laser fluence. This pulse number range is in agreement with the values found at fluences below the ablation threshold but above the melting threshold $[38,39]$. This result achieved for comparable number of laser pulses supports further that the melting of silicon governs the production of the fringed profiles and finally the production of microcones. 


\section{Conclusions}

The present work reports a systematic study of the interference patterning of a silicon surface with nanosecond laser pulses at $193 \mathrm{~nm}$ through a phase mask as a function of the laser fluence and of the number of pulses. Gratings with a spatial period of $1.7 \mu \mathrm{m}$ are obtained even upon irradiation with a single laser pulse when the fluence exceeds a value that allows silicon melting demonstrating that this patterning procedure is very fast compared to others. The topographic study together with the in-situ monitoring of the intensity of the first-order diffracted $\mathrm{cw}$ probe beam allow us to conclude that thermocapillary and/or Marangoni effects govern the process, inducing local material displacement as the silicon surface melts. This last statement is further supported by some recent results obtained by us on nanosecond single pulse modeling of surface modifications in silicon.

\section{Acknowledgements}

The authors gratefully acknowledge financial support from the MINECO (CTQ2016-75880-P). E.R. thanks MINECO for a Ramón y Cajal contract (RYC-2011-08069). Thanks are also given to T.A. Ezquerra for the use of the atomic force microscope. C.A.Z. thanks the Universidad Autónoma Metropolitana Iztapalapa and the Laboratorio de Supercómputo y Visualización en Paralelo for their support in the use of the YOLTLA cluster.

\section{References}

[1] Cai J and Qi L 2015 Recent advances in antireflective surfaces based on nanostructure arrays Mater. Horizons 2 37-53

[2] Haiyuan X, Sihua Z, Yufeng Z and Wenzhong S 2018 Controllable nanoscale inverted pyramids for highly efficient quasi-omnidirectional crystalline silicon solar cells Nanotechnology 29015403

[3] Carey J E, Crouch C H, Shen M and Mazur E 2005 Visible and near-infrared responsivity of femtosecond-laser microstructured silicon photodiodes Opt. Lett. 301773

[4] Küller A, Eck W, Stadler V, Geyer W and Gölzhäuser A 2003 Nanostructuring of silicon by electronbeam lithography of self-assembled hydroxybiphenyl monolayers Appl. Phys. Lett. 82 3776-8

[5] Fischer P B and Chou S Y 1993 Sub $\square 50$ nm high aspect $\square$ ratio silicon pillars, ridges, and trenches fabricated using ultrahigh resolution electron beam lithography and reactive ion etching Appl. Phys. Lett. 62 1414-6 
[6] Takagishi H, Masuda T, Yamazaki K and Shimoda T 2018 Fabrication of n-type Si nanostructures by direct nanoimprinting with liquid-Si ink AIP Adv. 8015214

[7] Li X and Bohn P W 2000 Metal-assisted chemical etching in $\mathrm{HF} / \mathrm{H} 2 \mathrm{O} 2$ produces porous silicon Appl. Phys. Lett. 77 2572-4

[8] Kelly J J and Philipsen H G G 2005 Anisotropy in the wet-etching of semiconductors Curr. Opin. Solid State Mater. Sci. 9 84-90

[9] de Boor J, Geyer N, Wittemann J V, Gösele U and Schmidt V 2010 Sub-100 nm silicon nanowires by laser interference lithography and metal-assisted etching Nanotechnology 21095302

[10] Guan Y F, Pedraza A J, Fowlkes J D and Joy D A 2004 Nanostructures produced by ultraviolet laser irradiation of silicon. II. Nanoprotrusions and nanoparticles J. Vac. Sci. Technol. B Microelectron. Nanom. Struct. 222836

[11] de Unamuno S and Fogarassy E 1989 A thermal description of the melting of c-and asilicon under pulsed excimer lasers Appl. Surf. Sci. 36 1-11

[12] Buividas R, Mikutis M and Juodkazis S 2014 Surface and bulk structuring of materials by ripples with long and short laser pulses: Recent advances Prog. Quantum Electron. 38 119-56

[13] Birnbaum M 1965 Semiconductor Surface Damage Produced by Ruby Lasers J. Appl. Phys. 36 36889

[14] Demchuk A V and Labunov V A 1995 Surface morphology and structure modification of silicon layers induced by nanosecond laser radiation Appl. Surf. Sci. 86 353-8

[15] Marcos Soldera, Kurt Taretto, Jana Berger and Andrés Fabián Lasagni 2016 Potential of Photocurrent Improvement in mc-Si:H Solar Cells with TCO Substrates Structured by Direct Laser Interference Patterning Advanced Engineering Materials 18 No. 9 1674-82

[16] Her T-H, Finlay R J, Wu C, Deliwala S and Mazur E 1998 Microstructuring of silicon with femtosecond laser pulses Appl. Phys. Lett. 73 1673-5

[17] Haro-Poniatowski E, Acosta-Zepeda C, Mecalco G, Hernández-Pozos J L, Batina N, Morales-Reyes I and Bonse J 2014 Diffraction-assisted micropatterning of silicon surfaces by ns-laser irradiation J. Appl. Phys. 115224309 
[18] Lu Y, Theppakuttai S and Chen S C 2003 Marangoni effect in nanosphere-enhanced laser nanopatterning of silicon Appl. Phys. Lett. 82 4143-5

[19] Brodoceanu D, Landström L and Bäuerle D 2007 Laser-induced nanopatterning of silicon with colloidal monolayers Appl. Phys. A 86 313-4

[20] Bonse J and Krüger J 2010 Pulse number dependence of laser-induced periodic surface structures for femtosecond laser irradiation of silicon J. Appl. Phys. 108034903

[21] Wang D, Wang Z, Zhang Z, Yue Y, Li D and Maple C 2013 Direct modification of silicon surface by nanosecond laser interference lithography Appl. Surf. Sci. 282 67-72

[22] Gedvilas M, Indrišiūnas S, Voisiat B, Stankevičius E, Selskis A and Račiukaitis G 2018 Nanoscale thermal diffusion during the laser interference ablation using femto-, pico-, and nanosecond pulses in silicon Phys. Chem. Chem. Phys., 2018, 20, 12166-12174

[23] Ihlemann J 2005 Ultraviolet laser ablation patterning of oxide films for optical applications Opt. Eng. 44051108

[24] Huster J, Muller J, Renner H and Brinkmeyer E 2011 Scanning Phase-Mask DUV Inscription of ShortPeriod Large-Area Photoresist Gratings J. Light. Technol. 29 2621-8

[25] Peláez R J, Afonso C N, Vega F, Recio-Sánchez G, Torres-Costa V, Manso-Silván M, García-Ruiz J P and Martín-Palma R J 2013 Laser fabrication of porous silicon-based platforms for cell culturing $J$. Biomed. Mater. Res. - Part B Appl. Biomater. 101 1463-8

[26] Solis J and Afonso C N 1991 Early stages of melting in Si under nanosecond laser pulse irradiation: A time-resolved study J. Appl. Phys. $692105-11$

[271] C. Acosta-Zepeda, P. Saavedra, J. Bonse, E. Haro-Poniatowski, accepted in JAP (2019)

[28] Abeles B, Beers D S, Cody G D and Dismukes J P 1962 Thermal Conductivity of Ge-Si Alloys at High Temperatures Phys. Rev. 125 44-6

[29] Glassbrenner C J and Slack G A 1964 Thermal Conductivity of Silicon and Germanium from $3^{\circ} \mathrm{K}$ to the Melting Point Phys. Rev. 134 A1058-69

[30] http://kjinnovation.com/GD-Calc.html (cited on the 29'th of August 2018)

[31] Philipp H R and Ehrenreich H 1963 Optical Properties of Semiconductors Phys. Rev. 129 1550-60 
[32] Millot F, Sarou-Kanian V, Rifflet J-C and Vinet B 2008 The surface tension of liquid silicon at high temperature Mater. Sci. Eng. A 495 8-13

[33] Cazabat A M, Heslot F, Troian S M and Carles P 1990 Fingering instability of thin spreading films driven by temperature gradients Nature $\mathbf{3 4 6} 824-6$

[34] Lowndes D H, Fowlkes J D and Pedraza A J 2000 Early stages of pulsed-laser growth of silicon microcolumns and microcones in air and SF6 Appl. Surf. Sci. 154-155 647-58

[35] Sánchez F, Morenza J L, Aguiar R, Delgado J C and Varela M 1998 Dynamics of the hydrodynamical growth of columns on silicon exposed to ArF excimer-laser irradiation Appl. Phys. A Mater. Sci. Process. $6683-6$

[36] Sarnet T, Carey J E and Mazur E 2012 From black silicon to photovoltaic cells, using short pulse lasers pp 219-28

[37] Zheng B, Wang W, Jiang G, Wang K and Mei X 2015 Research status and application prospects of manufacturing technology for micro-nano surface structures with low reflectivity Proc. Inst. Mech. Eng. Part B J. Eng. Manuf. 229 1877-92

[38] Zuev D A, Novodvorsky O A, Khaydukov E V., Khramova O D, Lotin A A, Parshina L S, Rocheva V V., Panchenko V Y, Dvorkin V V., Poroykov A Y, Untila G G, Chebotareva A B, Kost T N and Timofeyev M A 2011 Fabrication of black multicrystalline silicon surface by nanosecond laser ablation Appl. Phys. B 105 545-50

[39] Sánchez F, Morenza J L, Aguiar R, Delgado J C and Varela M 1996 Whiskerlike structure growth on silicon exposed to ArF excimer laser irradiation Appl. Phys. Lett. 69 620-2 


\section{FIGURE CAPTIONS}

FIGURE 1: Experimental set-up for phase mask laser interference irradiation. The induced transformation has been followed by measuring the first diffraction order by means of a probe HeNe laser beam.

FIGURE 2: Photos of the laser irradiated Si sample taken with natural light illumination under slightly different angles of view; the coloured areas correspond to the laser irradiated regions and the grey areas are the non- irradiated regions in the Si sample. All gratings have the 1D fringe patterns along the horizontal direction and the camera is slightly moving in the vertical direction around the first diffraction order.

FIGURE 3: (a) First order diffraction intensity at $\lambda=632.8 \mathrm{~nm}$ as a function of number of laser pulses at three different laser fluences $\left(630,830,1000 \mathrm{~mJ} \mathrm{~cm}^{-2}\right)$, where four different stages (I IV) have been identified (see text). (b) Detailed evolution of the diffraction signal during the first laser pulses. The lines guide the eye. The intersections of these lines define the borders between adjacent process stages I and II. (c) Pulse number span of the four different stage.

FIGURE 4: Optical micrographs of surface patterns produced at increasing number of laser pulses from top to bottom, the labels are the number of laser pulses and the laser fluence is $1000 \mathrm{~mJ} \mathrm{~cm}^{-}$ 2.

FIGURE 5: (a) Normalized surface reflectivity profiles of representative period, extracted from the horizontal lines in Fig. 4 ( $x$ is given in units of the spatial period $\Delta$ ). These relative reflective signals are referred to the reflectivity of the non-irradiated crystalline Si. The number of laser pulses, $(\#=1-2000)$, increases from bottom to top. For better visibility, the reflectivity profiles are shifted in the vertical direction by $1.3 \%$. (b) $2 \mathrm{D}$ representation of these profiles in a color map.

FIGURE 6: AFM topography images of surface patterns produced at a fluence of $1000 \mathrm{~mJ} \mathrm{~cm}^{-2}$, the labels indicate the number of pulses. 
FIGURE 7: (a) Depth-profiles of representative periods that have been obtained from the topography images provided in Fig. 6. For better visibility, the depth profiles are shifted in the vertical direction by $7 \mathrm{~nm}$. The number of laser pulses, \#, increases from bottom to top. (b) 2D representation of these profiles where the mean height in vertical direction has been set to zero. FIGURE 8: Parameters obtained from the AFM depth profiles and optical signals as a function of the number of laser pulses (\#) in $\log$ scale for a fluence of $1000 \mathrm{~mJ} \mathrm{~cm}^{-2}$ : (a) (•) surface height modulation, $z$, (see text for definition) obtained from the AFM depth profiles of Fig. 7a, (O) optical contrast, $C$, obtained from the optical reflectivity profiles of Fig. 5a and (solid line) first order diffraction signal (mV) extracted from Fig. 3 (b) (घ) Width and asymmetry, A,( $\square$ ) of the profiles show in Fig. 6a. See text for description of $z, C$ and $A$. (c) First order diffraction efficiency simulated by using the parameters $(z, C, A)$ shown in a) and b) ( $\mathbf{\Delta})$ and measured (solid line).

FIGURE 9: Comparison of the surface topography generated with a single laser pulse measured with AFM (blue line) and a surface modification computed (green line) with our model.

FIGURE 10: (a) Optical (b) AFM and (c) cross-section SEM images of the irradiation produced for 5000 laser pulses for a fluence of $1000 \mathrm{~mJ} \mathrm{~cm}-2$. Scale bar is the same in all images. 
FIGURE 1

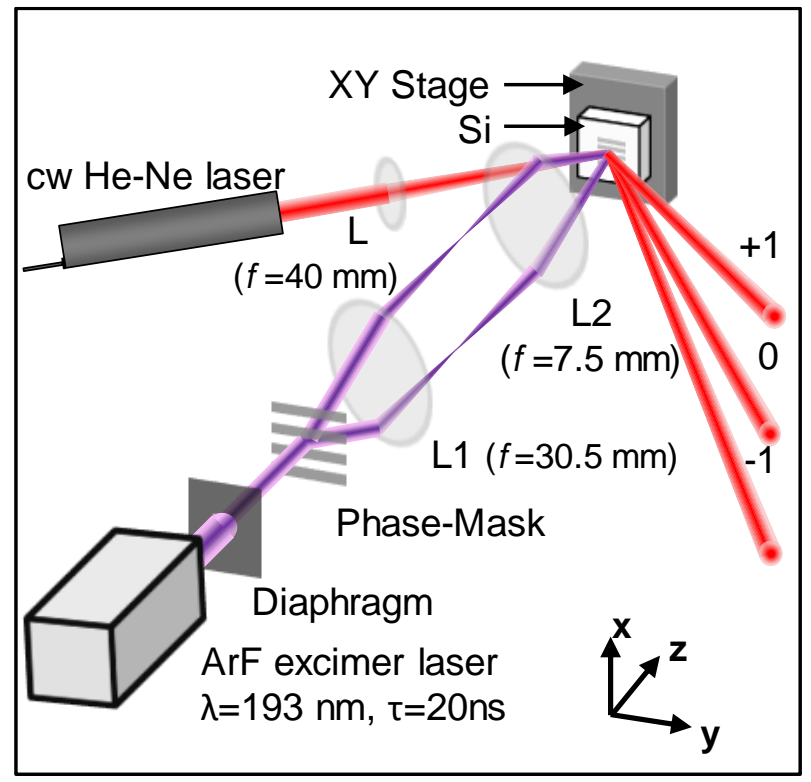


FIGURE 2

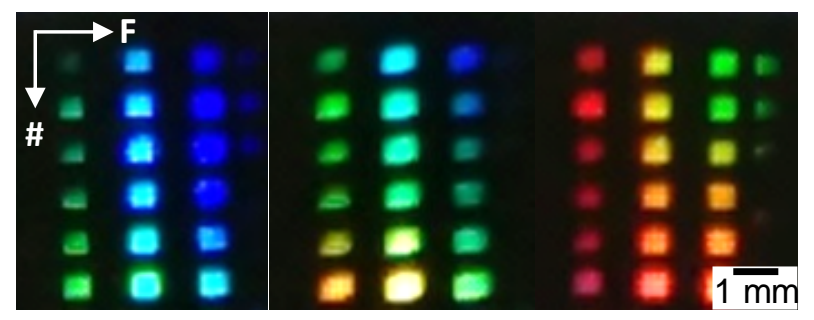


FIGURE 3
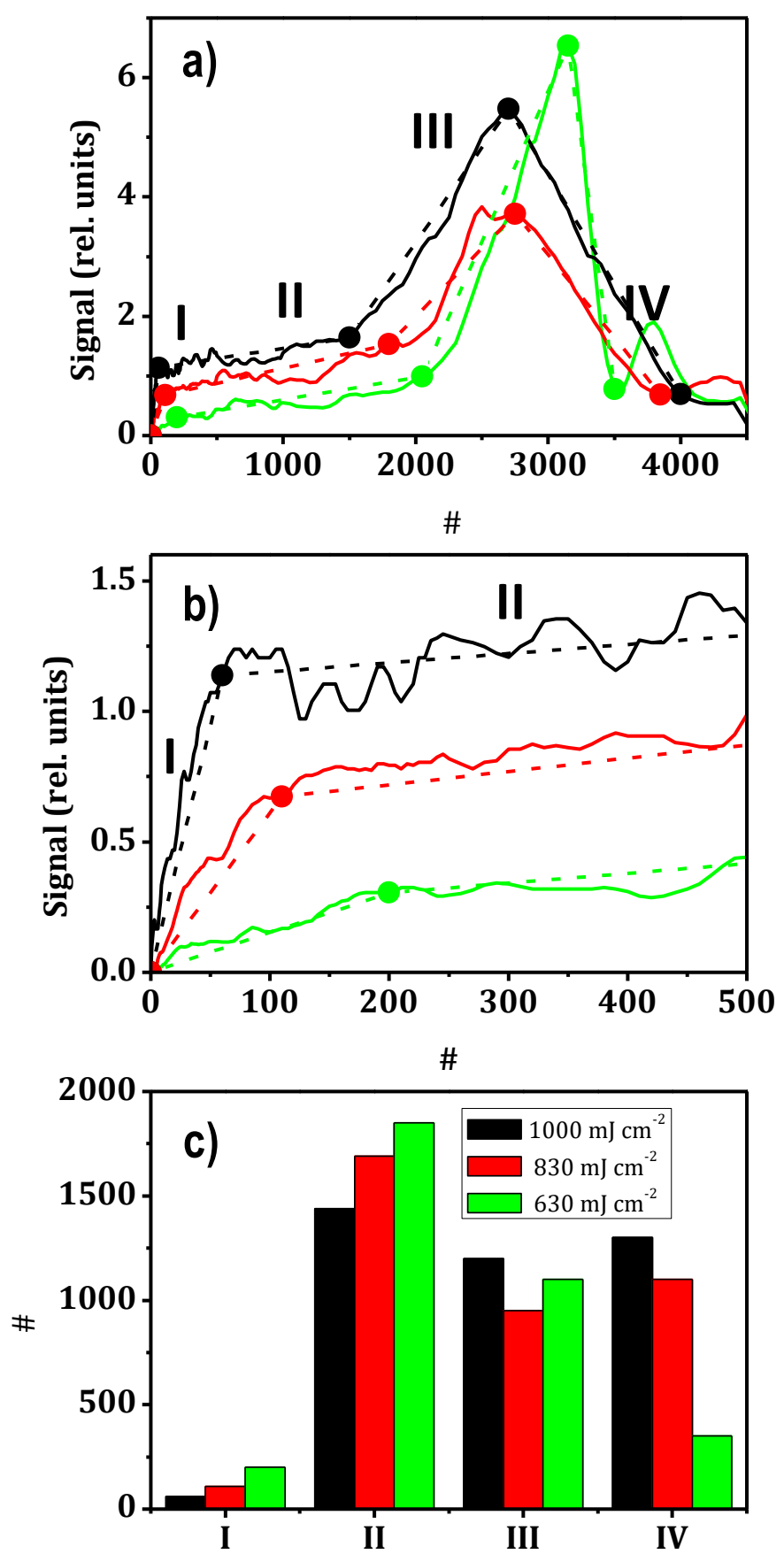
FIGURE 4

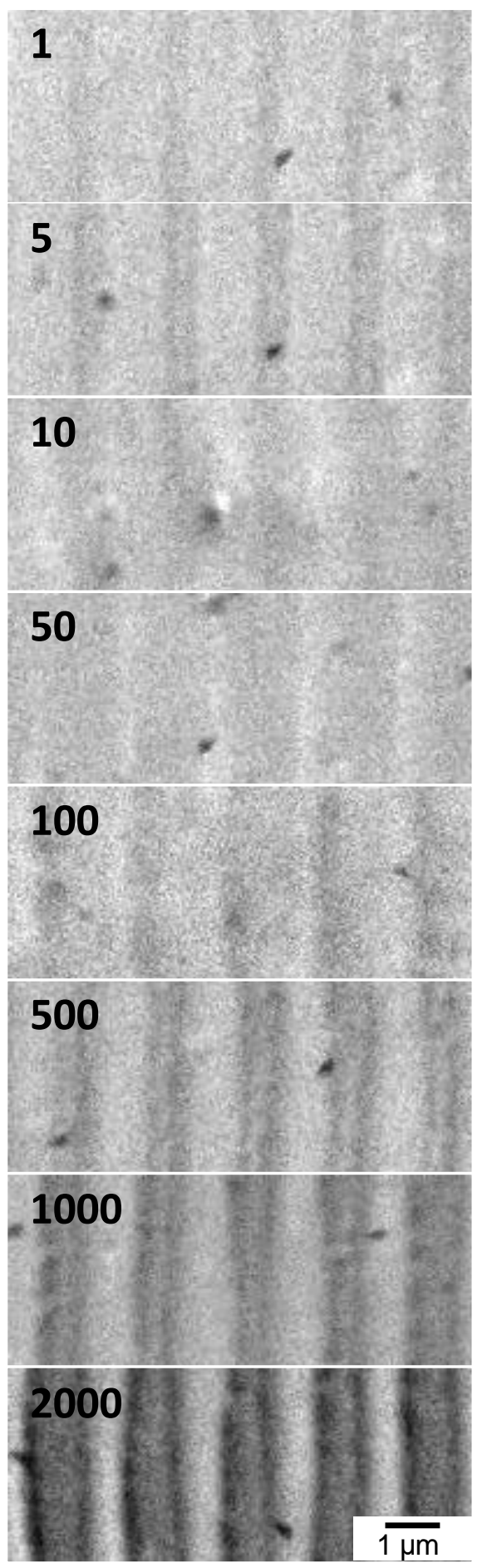


FIGURE 5

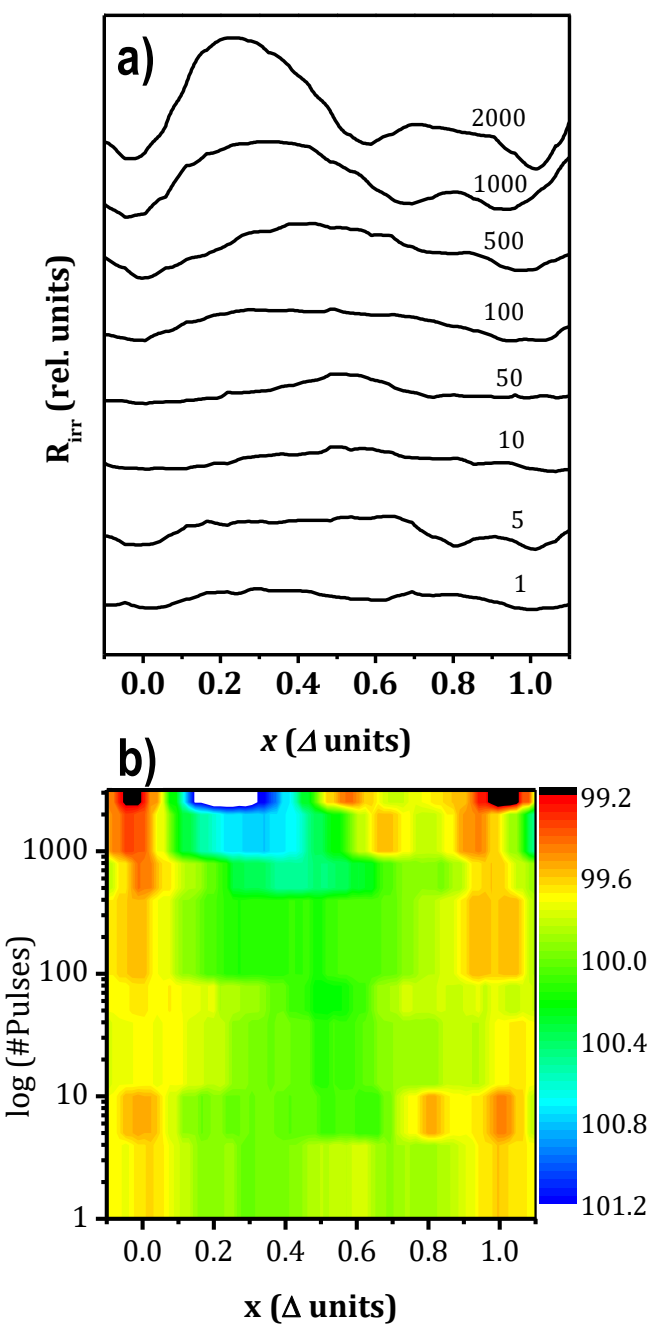


FIGURE 6

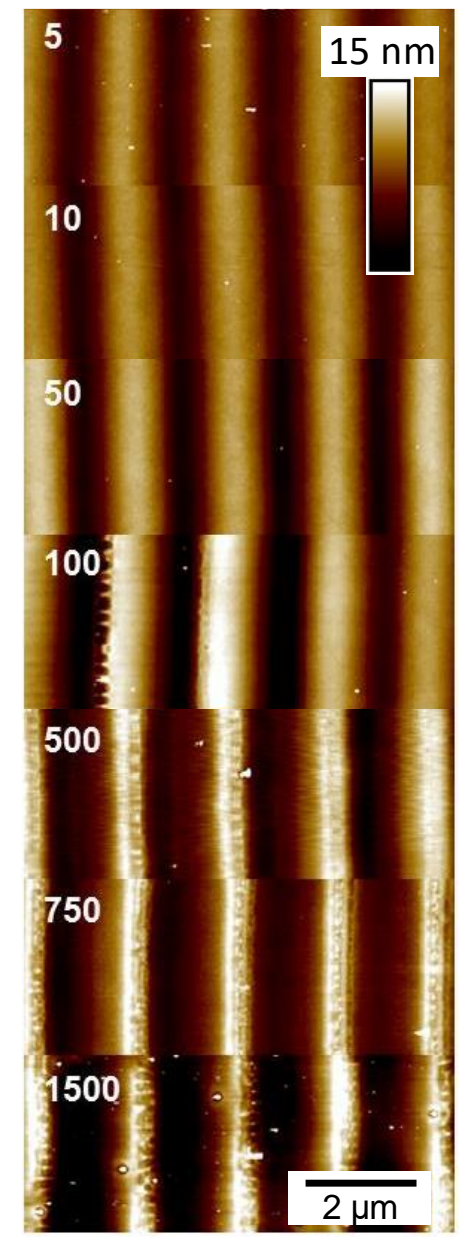


FIGURE 7
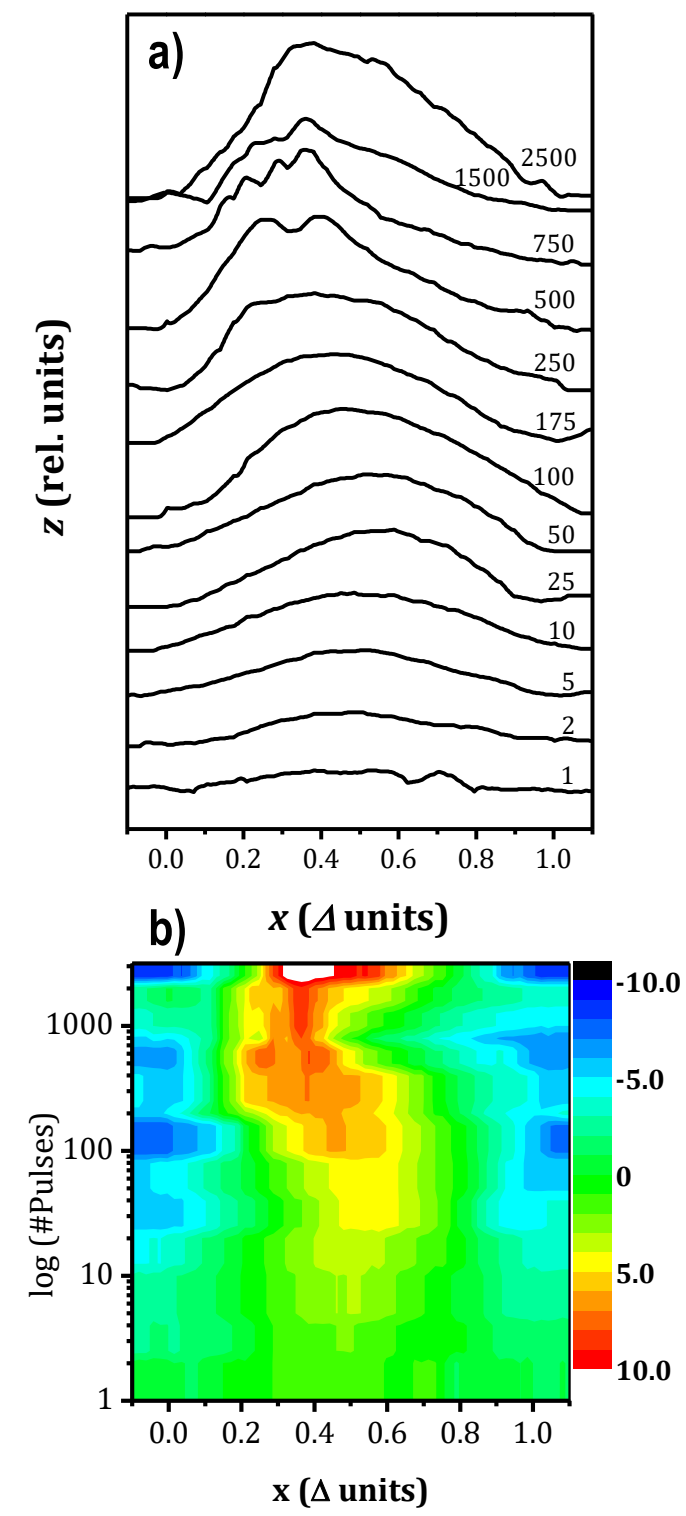
FIGURE 8

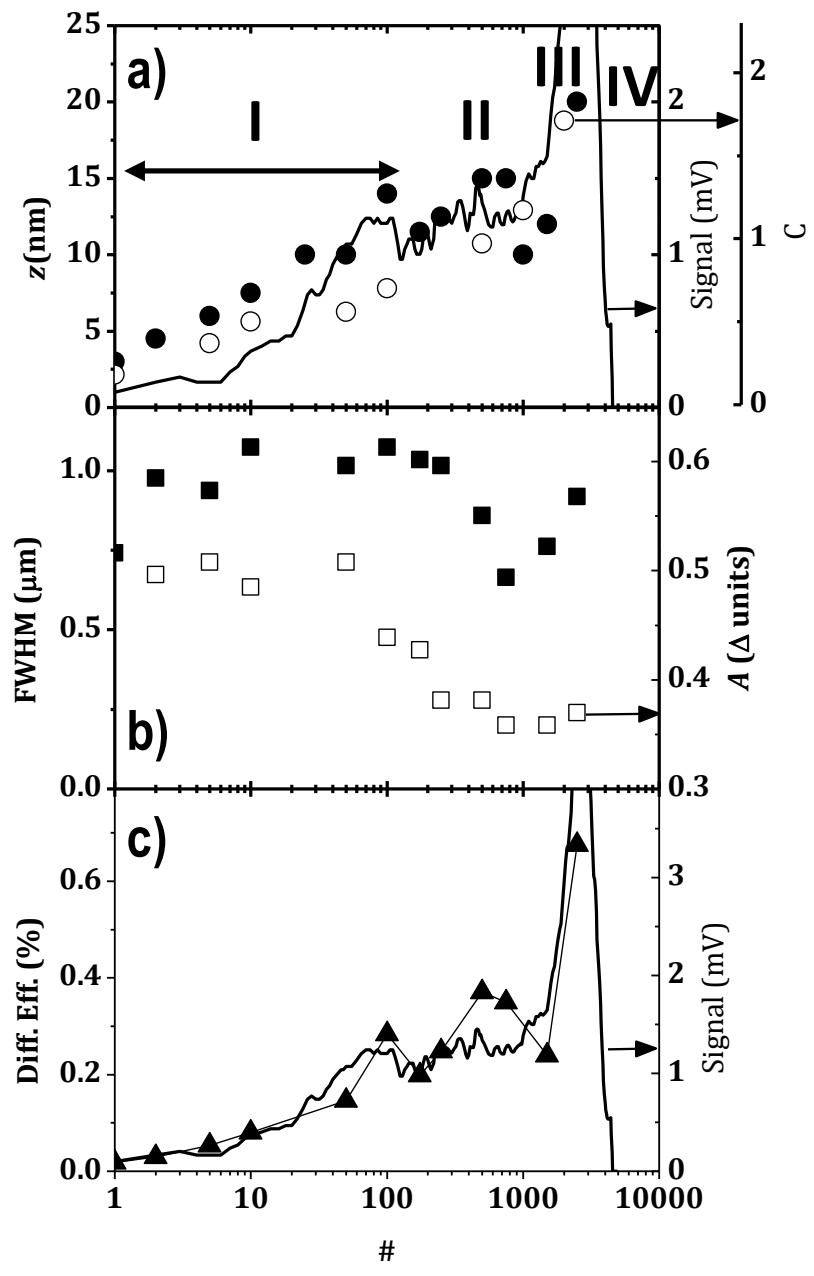


FIGURE 9

हัง
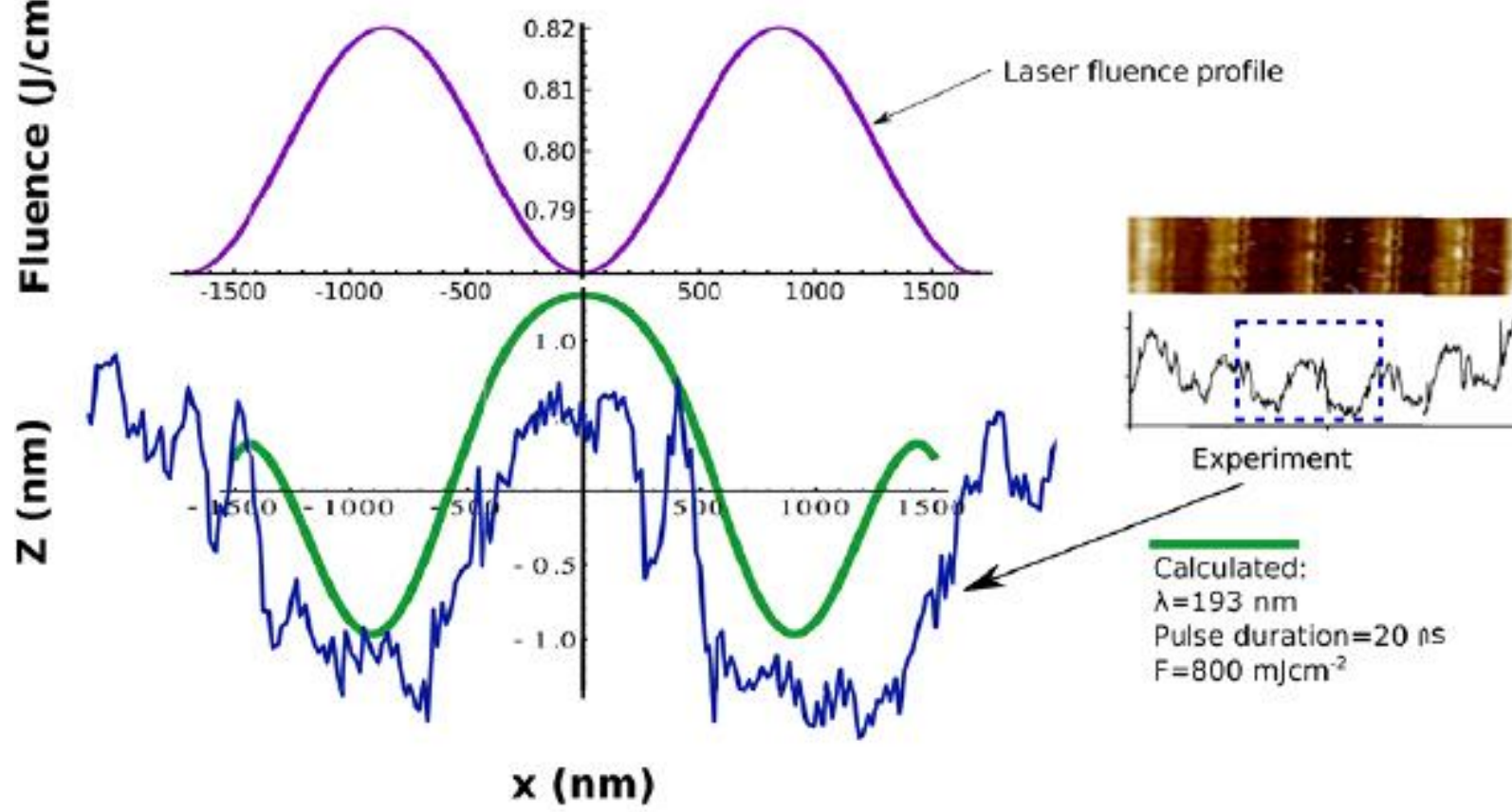
FIGURE 10

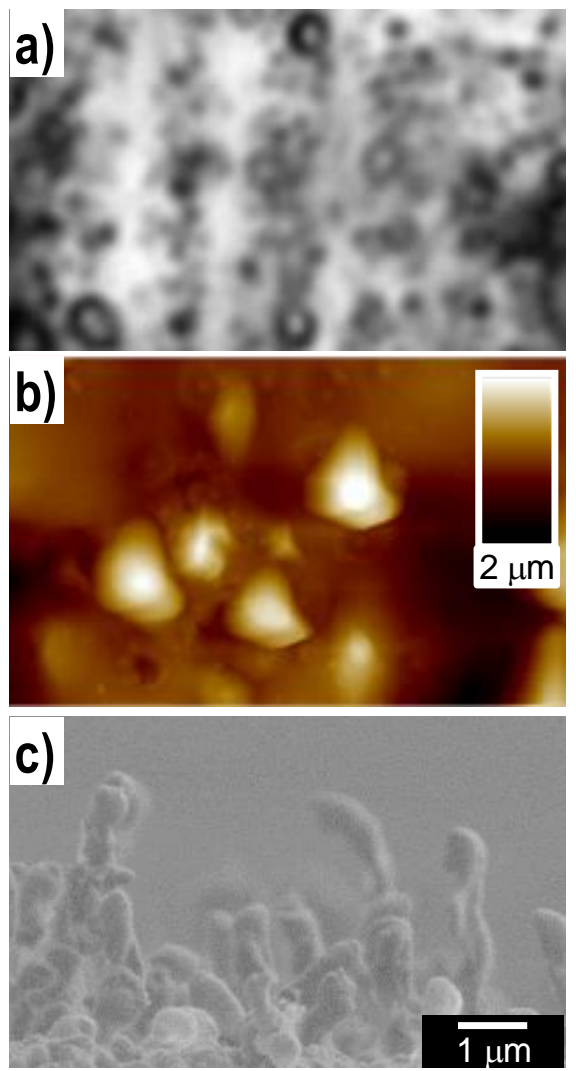

